\title{
Spatial Pattern Analysis and Sequential Sampling for the Incidence of Leaf Spot on Strawberry in Ohio
}

\author{
W. W. Turechek and L. V. Madden, Department of Plant Pathology, The Ohio State University, Ohio Agricultural \\ Research Development Center, Wooster 44691
}

\begin{abstract}
Turechek, W. W., and Madden, L. V. 1999. Spatial pattern analysis and sequential sampling for the incidence of leaf spot on strawberry in Ohio. Plant Dis. 83:992-1000.

Spatial pattern of the incidence of strawberry leaf spot, caused by Mycosphaerella fragariae (Ramularia brunnea), was quantified on commercial strawberry farms in Ohio. For each planting of strawberry, one or two transects were randomly chosen, and the proportion of leaflets (out of 15) with leaf spot was determined from $N=29$ to 87 evenly spaced sampling units. Based on a likelihood ratio test, the beta-binomial distribution described the frequency of diseased leaflets better than the binomial in $93 \%$ of the 59 data sets over 3 years. Estimates of mean incidence ranged from 0.0009 to 0.82 , with a median of 0.05 . Estimates of the beta-binomial aggregation parameter, $\theta$, ranged from 0 to 1.06 , with a median of 0.20 . Moreover, the estimate of the slope of the binary power law, fitted to the variance data for the 59 data sets, was significantly $(P<$ $0.01)$ greater than one, indicating that heterogeneity, and hence the pattern of disease incidence at the spatial scale of the sampling units or smaller, was dependent on mean incidence. Spatial autocorrelation and Spatial Analysis by Distance IndicEs (SADIE) analyses detected significant positive association of disease incidence among sampling units in approximately $40 \%$ of the data sets, indicating that disease clusters extended beyond the borders of the sampling units in these fields. Collectively, the results show that strawberry leaf spot was characterized by relatively tight clusters of disease (based on $\theta$ ) that extended beyond the borders of the sampling units in a little under half of the data sets (based on correlations). The information on heterogeneity was used to develop fixed and sequential sampling curves to precisely estimate disease incidence. The sequential-estimation procedure was evaluated using statistical bootstrap methods and performed well over the range of disease incidences encountered.
\end{abstract}

Strawberry leaf spot is one of the most common and destructive diseases of cultivated strawberry (Fragaria $\times$ ananassa) worldwide (9). The causal agent is the fungus Mycosphaerella fragariae (anamorph Ramularia brunnea, syn. $R$. tulasnei). The pathogen primarily infects young, expanding leaves and petioles; however, a serious disease of strawberry known as black seed occasionally occurs when this pathogen infects the fruit. Typical disease development begins in late spring or early summer in northern U.S. states. The fungus overwinters as mycelia, sclerotia, and/or perithecia in infected leaf tissue. Ascospores, which typically serve as a source of primary inoculum, are discharged from perithecia in early spring and are disseminated by wind. The major source of both primary and secondary inoculum throughout the growing season, however, is thought to be the conidia originating in diseased leaf tissue and sclerotia (9). Although it has been stated that conidia are

Corresponding author: L. V. Madden

E-mail: MADDEN.1@osu.edu.

Accepted for publication 13 July 1999.

Publication no. D-1999-0817-01R

(C) 1999 The American Phytopathological Society splash dispersed $(4,9)$, this has not been demonstrated. Secondary cycles can occur repeatedly under favorable conditions. Leaf spot can be severe in regions where strawberries are grown perennially because inoculum can build up substantially over the years.

Because of the sporadic nature of the disease, routine scouting of strawberry fields may be helpful in determining disease incidence and predicting whether a fungicide is needed (2). However, surveys of strawberry fields can be time-consuming and potentially costly. Surveying could be greatly facilitated by an efficient sampling program in which the number of sampling units assessed for disease symptoms is based on mean incidence and the variability of disease incidence in a field, the latter being a function of spatial pattern of disease (2). An efficient sampling plan will not only serve in determining the immediate status of disease incidence in a field, but may also provide the reliable epidemiological information needed to develop a forecaster or other controls. However, information needed to develop sampling plans is lacking. In particular, no information is available on the spatial pattern of leaf spot.

Many statistical procedures are available to quantify spatial patterns of disease. Spatial point-pattern analyses and geostatistics are two general classes of statistical procedures that have been extensively used in botanical epidemiology for analysis of disease patterns. Spatial point-pattern techniques aim to quantify disease pattern at the level of the sampling unit and below, or the pattern of diseased individuals within sampling units (20). Fitting statistical distributions to counts of diseased individuals per sampling unit is an example of a pointpattern approach used to quantify pattern (heterogeneity). Geostatistical techniques are used to quantify pattern at the level of the sampling unit and above, or among sampling units. Autocorrelation analysis is a method that belongs to this category. A new correlation-based method was recently developed called Spatial Analysis by Distance IndicEs (SADIE) $(15,16)$. An interesting feature of SADIE is that results are conditioned on the heterogeneity of the data set, thus allowing one to build upon the results of a point-pattern analysis. Collectively, these two general approaches (i.e., point-pattern and geostatistics) can jointly be used to determine the average size of disease clusters and the degree of aggregation within disease clusters.

The purpose of this research was to: (i) quantify the spatial pattern (i.e., heterogeneity and spatial arrangement) of the incidence of strawberry leaf spot in perennial production systems in Ohio and relate this to mean disease incidence; (ii) determine if spatial pattern was associated with crop conditions, such as cultivar, assessment time, and farm location; and (iii) determine the number of sampling units required to estimate disease incidence per field with a desired level of precision.

\section{MATERIALS AND METHODS}

Data collection. Naturally occurring epidemics of strawberry leaf spot were monitored over 3 years. In 1996 and 1997, leaf spot was assessed at a single commercial strawberry farm in Ohio, located approximately $22 \mathrm{~km}$ southwest of Columbus in Darbydale $\left(39^{\circ} 51^{\prime} 13^{\prime \prime} \mathrm{N}, 83^{\circ} 10^{\prime} 59^{\prime \prime} \mathrm{W}\right)$. The farm had several perennial plantings (fields) comprised of 10 to 20 rows of strawberry. The plantings ranged in age from 1 to 5 years. Plants were grown in a matted-row system approximately $0.3 \mathrm{~m}$ wide on 1-m centers. Cultivars Earliglow and Honeoye were the dominant ones grown; other cultivars rated for disease included Delmarvel and Primetime.

In 1996, sampling started on 13 July and continued until 13 September. In 1997, 
sampling started on 29 April and continued until 26 September. Sampling occurred at approximately biweekly intervals in both years. These dates encompassed time of fruit development, harvesting, renovation, and regrowth of vegetation. Three or four fields were randomly selected for disease assessment from a total of eight or nine fields during each sampling date over the 2 years. Not all fields were sampled the same number of times. The same leaves were not necessarily sampled in different assessments of the same field.

In 1998, a statewide survey of foliar diseases of strawberry on 26 commercial farms was conducted from 16 June through 23 June. This time was chosen to coincide with harvesting. Farms selected for the survey were chosen in a stratified random sample. In this survey, the state was divided into nine regions or strata (Fig. 1), and commercial strawberry farms within these regions were identified from the member directories of the National Association of Strawberry Growers and the Ohio Small Fruit Growers Association. Additional farms were identified through personal communications with growers. From the compiled listing, three or four farms were randomly chosen from each of the nine regions or strata (except in regions 8 and 9, where only two and one strawberry farms could be identified, respectively; Fig. 1).

On most farms, separate fields were clearly distinguishable, and on other farms, arbitrary field demarcations were imposed. On farms where fields were not clearly defined, ad hoc fields were created based on cultivar. For each farm, three fields were randomly chosen from the fields identified (ad hoc or other) for disease assessment.

Total area devoted to strawberry production on these 26 farms ranged from approximately 14 ha to less than 1 ha. The perennial plantings ranged in age from 1 to 7 years. Cultivars assessed for disease included Earliglow, Honeoye, Jewel, Allstar, Red Chief, Sure Crop, Cavendish, and Noreaster. Horticultural practices varied tremendously among farms. Most farms used a matted-row system. However, one farm used a split-row system, and a few farms had very poorly managed fields. Weed control on most farms was incomplete, while others had excellent weed control, and a few had fields hardly identifiable as strawberry plantings due to the weeds.

Fields were sampled almost identically over the 3 years. For each field and assessment time, a single row was randomly chosen as the primary transect. In 1996 and 1997, a short secondary transect was arbitrarily chosen to approximately bisect the field; this transect was not included in 1998 samplings. Sampling units consisted of 15 leaflets (five leaves of three leaflets each). Each leaflet was rated for the presence or absence of leaf spot symptoms.
This is a form of cluster sampling in which the clusters (i.e., sampling units) were chosen systematically and all individuals (e.g., leaflets) per sampling unit $(n)$ were assessed (8). The initial sampling unit was arbitrarily chosen within the first 20 steps of the primary transect, and each subsequent sampling unit was located at a distance of three steps $(\sim 2.5 \mathrm{~m})$ from the previous sampling unit, assuring approximately evenly spaced sampling units. In 1996 and 1997, a sampling unit in each row also was sampled along the secondary (bisecting) transect. The number of sampling units, $N$, varied from 27 to 87 , depending on field size, but was typically between 65 and 75 .

In 1996 and 1997, 46 independent data sets (field assessments) were collected from the single farm in Darbydale. Thirty of these data sets contained leaf spot data. In 1998, 16 of the 26 farms surveyed had fields with leaf spot, primarily in the southern half of the state. Of the possible 48 fields (16 farms $\times 3$ fields), 29 had leaf spot. In total, 124 data sets were collected from 1996 to 1998 ; 59 of these were used to quantify the spatial pattern of leaf spot.

Point-pattern approaches. Distribution and indices analysis. The beta-binomial $(7,11)$ and the binomial distributions were fitted to the data using BBD (10) for each individual field assessment. The binomial distribution has a single parameter representing the probability of disease $(\pi)$. The beta-binomial has two parameters: $p$, which is the expected probability of disease; and $\theta$, which is a measure of variation in disease incidence per sampling unit. A good fit to the binomial distribution is suggestive of a random spatial pattern of disease incidence, while a good fit to the beta-binomial is suggestive of an aggregated pattern of disease incidence. Standard chi-square goodness-of-fit tests (7) were calculated for each distribution after disease incidence classes (i.e., 0 to 15 diseased leaflets) were pooled so that all expected frequencies exceeded five. The degrees of freedom (df) are given by: number of disease classes after pooling number of parameters - 1 . Maximum likelihood as well as moment estimates of the beta-binomial parameters, were calculated. When it was not possible to obtain maximum likelihood estimates, moment estimates were used in assessing goodness-offit. A log-likelihood ratio test statistic (LRS) was calculated for each data set to determine if the beta-binomial fitted the data better than the binomial (21). LRS has a $\chi^{2}$ distribution with one degree of freedom under the null hypothesis of no difference between the two log-likelihoods (i.e., when the beta-binomial does not characterize the frequency distribution of diseased leaflets or leaves any better than the binomial) (21).

Binary power law analysis. The binary form of Taylor's power law (19) can be used to assess the degree of heterogeneity in a collection of data sets. The binary power law, as written by Hughes and Madden (6), can be expressed as a relationship between two variances: the observed sample variance of diseased leaflets $\left(v_{\mathrm{obs}}\right)$ and the theoretical variance of a random distribution $\left(v_{\text {ran }}\right)$

$v_{\mathrm{obs}}=A_{x} v_{\mathrm{ran}}^{b}$

in which $A_{x}$ and $b$ are parameters. With binary data, $v_{\text {ran }}$ is the binomial variance,

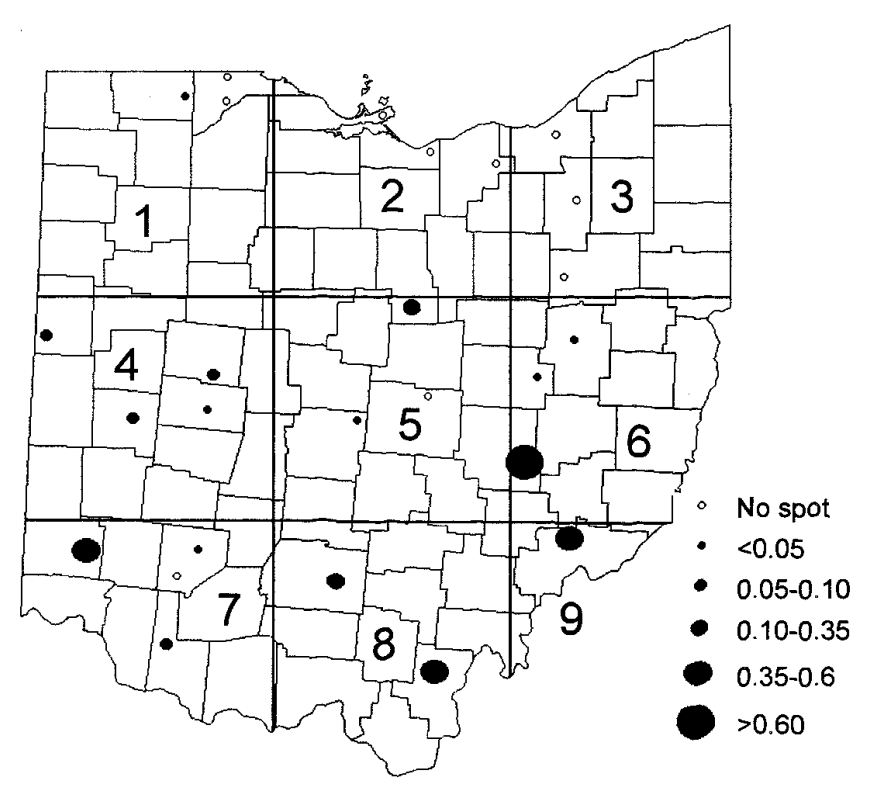

Fig. 1. Map of Ohio showing the locations of the 26 strawberry farms surveyed in 1998. Each circle represents average disease incidence from three randomly chosen fields at that farm location. Solid circles indicate presence of leaf spot at that location, with larger circles representing greater disease incidence (see label). Open circles correspond to no disease. 
$n p(1-p)$, in which $n=15$ (leaflets per sampling unit) here. By taking logarithms of both sides of equation 1 , we obtain

$\ln \left(v_{\text {obs }}\right)=\ln \left(A_{x}\right)+b \ln [n p(1-p)]$

where $\ln \left(A_{x}\right)$ and $b$ are the intercept and slope of a straight line. In fitting equation 2 to the data by regression analysis, we used the estimated $p$ from the beta-binomial distribution $(\hat{p})$ and the moment estimate of $v_{\text {obs }}$ for each data set. When $A_{x}$ and $b$ are both equal to 1 , randomness as described by the binomial distribution is indicated. When $b=1$ and $A_{x}>1$, there is overdispersion, but the degree of overdispersion for each data set (e.g., $\theta$ of the beta-binomial) does not depend on $p$. When $b$ and $A_{x}$ are both greater than 1 , the degree of overdispersion or heterogeneity changes with $p$. The beta-binomial parameter $\theta$ can be written in terms of the power law estimates (11):

$\theta=[a-f(p) / n] /[f(p)-a]$

where $f(p)=[p(1-p)]^{1-b}$ and $a=A_{x} n^{b-2}$ $(11,20)$. Equation 3 represents a curve with maximum value at $p=0.5$ when $b>1$ and a horizontal line when $b=1$. The relationship between $\theta$ and $p$ is important for characterizing the spatial process and for sampling (11).

For power-law analysis, the data sets of 1996 and 1997 were combined, because the number of individual data sets in 1996 or 1997 alone was too small to allow precise parameter estimation in the regression analysis. In total, 30 data sets were obtained in 1996 and 1997 and 29 in 1998.

A covariance analysis was conducted to determine the effect of the factors (class variables) cultivar, assessment time (for 1996 and 1997 data set only), region of state (for 1998 data set only), and year (all data) on the estimated slope $(b)$ and/or intercept $\left[\ln \left(A_{x}\right)\right]$ of the binary power law (eq. 2) (3). Covariance methods were the same as in Madden et al. (12) and Turechek and Madden (20). GLIM (Generalized Linear Interactive Modelling, version 3.77, Oxford, London) was used for the regression analysis. The continuous predictor variable $\ln [n \hat{p}(1-\hat{p})]$ was always included in the model first. Factors were then added, first as an intercept only term, and then as a slope term to determine a factors effect on $\ln \left(A_{x}\right)$ or $b$, respectively. A factor was considered to significantly contribute to observed heterogeneity if the new model's deviance was significantly lower than the deviance of the original power law model. Because the binary power law is based on the principle that heterogeneity $\left(v_{\mathrm{obs}}\right)$ is related to disease incidence (11), the test of a factor in the covariance analysis is not an indication of whether or not the factor had a direct influence on disease incidence or on heterogeneity, but on whether or not the factor affected the degree of heterogeneity after first correcting for the relationship between heterogeneity and $p$. The covariance analysis for cultivar, assessment time, and region was first done for each year category (1996-97 and 1998) separately; then a separate covariance analysis was done to determine if the year factor affected $\ln \left(A_{x}\right)$ or $b$ using the combined data set (i.e., 1996-97 and 1998).

Geostatistical-type approaches. Spatial autocorrelation. For each individual field assessment, the number of diseased leaflets in each sampling unit along the primary transect was transformed using the logit transformation, $\ln [y /(1-y)]$, where $y=(x$ $+0.5) /(n+1)$ and $x$ is the number of diseased leaflets in each sampling unit containing $n$ leaflets. This corrected value of disease proportion, known as the Haldane correction $(17,18)$, was used to avoid taking the logarithm of zero values or dividing by 0 . Spatial autocorrelation coefficients were calculated for the standard transect analysis with the statistical program Minitab (Minitab, version 10, State College, PA). The secondary transects of 1996 and 1997 (i.e., the short transects of sampling units across fields) were not used in the spatial autocorrelation analysis.

Spatial Analysis by Distance IndicEs (SADIE). For each data set, the SADIE program was used to calculate the distance to regularity $\left(D_{r}\right)(15,16,20)$ using data from the primary transect. Regularity is defined as the condition when each individual sampling unit contains the mean number of individuals per field (i.e., each sampling unit contains $\Sigma X / N$ diseased individuals). SADIE employs an algorithm that produces a regular arrangement of counts from any lattice of spatially referenced data by moving individuals (diseased leaflets) from one location (sampling unit) to another via the shortest possible route (distance). Summing the distances each individual moved in order to achieve regularity gives the distance to regularity. A randomization test was used to determine if the observed distance to regularity is a particularly large or small value. To perform this test, SADIE randomly rearranges the sampling-unit counts and, from this new arrangement, calculates a new distance to regularity. For each data set, this was done 100 times to derive a frequency distribution of distances to regularity. From this frequency distribution, percentiles were calculated, and the significance level for a one-sided test for aggregation was determined from the proportion of randomizations with distances to regularity as great or greater than the distance for the observed data $\left(D_{r}\right)$. Because the SADIE test uses the rearrangement of observed data, results are conditioned on the degree of heterogeneity of the data.

An index of aggregation $\left(I_{a}\right)$ based on the SADIE methodology can be written: $I_{a}$ $=D_{r} / E_{a}$, in which $E_{a}$ is the average (expected) distance to regularity of the 100 randomization results. A value of $I_{a}=1$ suggests a spatially random pattern; $I_{a}>1$ suggests a more aggregated pattern; and $I_{a}$ $<1$ suggests a more regular pattern. However, $I_{a}$ is not directly used for determining significance; only the proportion of distances from the randomizations is used. There are other types of tests in SADIE (e.g., distance to clustering) that are not relevant to binary data such as disease incidence.

Sampling curves. Sampling curves were developed using the results of the binary power law so that incidence of leaf spot could be estimated with a specified level of precision $(8,13)$. In our situation, precision was determined with the coefficient of variation of the mean, $C$, defined as the standard error of estimated $p(\operatorname{SE}[\hat{p}])$ divided by $\hat{p}(8,12)$. The standard error of $\hat{p}$ is found using: $\sqrt{ } a[\hat{p}(1-\hat{p})]^{b} / N$ (8). This expression characterizes the variance of $\hat{p}$ when disease incidence has a beta-binomial distribution and when aggregation (as characterized by $\theta$ ) varies systematically with incidence in a field $(p)$ (as summarized from the binary power law). The number of sampling units $(N)$ required to estimate $p$ with reliability defined by $C$ is:

$N=a p^{b-2}(1-p)^{b} / C^{2}$

where $a, b, p$, and $C$ are as defined above. Use of equation 4 is equivalent to defining $\operatorname{SE}(\hat{p})$ as a function of $\theta$, with $\theta$ changing in relation to $p(8)$. In practice, equation 4 is most useful when the investigator has a general idea of the magnitude (level) of $p$ before sampling. However, using $p=0.5$ will maximize equation 4 , thus assuring that the precision of $\hat{p}$ will be no worse than that of $C$, and typically will be less than $C$ if the binary power law represents the data.

An alternative method for estimating $p$ when $p$ is unknown is the method of sequential estimation (13). Here the total number of diseased leaflets, $T_{N}$, is tallied after each sampling unit, where $T_{N}=\Sigma x_{i}$ and $x_{i}$ is the number of diseased leaflets in the $i$ th sampling unit. When $T_{N}$ exceeds some threshold (critical) value, called the stop limit, determined by $C$ in our case, sampling ceases; $p$ can then be easily determined using $p=T_{N} / n N$. When $b>1$ in the binary power law, one cannot write $T_{N}$ as a simple function of $a, b, p$, and $C$ (13). However, it can be written as a function of $T_{N}\left[\gamma_{N}\right]$, which can then be solved numerically for $T_{N}$. The function is written as:

$\gamma_{N}=T_{N}^{b-2}\left(n N-T_{N}\right)^{b}=\left(\frac{C^{2}}{a}\right) n^{2 b-2} N^{2 b-1}$

A Mathcad (Mathsoft, Inc., Cambridge, MA) worksheet was written to iteratively solve the $\gamma_{N}$ equation for $T_{N}$ at $N=1$ to 500 and $C=0.2,0.3$, and 0.4 .

Sequential sampling evaluation. Sequential-estimation sampling was simulated from a subset of the 59 data sets using statistical bootstrap methods to evaluate the performance of the sequential- 
estimation procedure. Methods were based on, and fully described in, Madden et al. (13). The 59 data sets were divided into six categories based on their incidence of leaf spot. The following categories were constructed: $0.01 \leq \hat{p}<0.05 ; 0.05 \leq \hat{p}<0.1$; $0.1 \leq \hat{p}<0.2 ; 0.2 \leq \hat{p}<0.4 ; 0.4 \leq \hat{p}<0.65$; $\hat{p} \geq 0.65$. From each category, three data sets were randomly chosen, except for the fifth category $(0.4 \leq \hat{p}<0.65)$, where only three data sets were available. The stop line (i.e., values of $T_{N}$ ) was generated using equation 5 , with reliability defined by the coefficient-of-variation and values of $a$ and $b$ estimated for the pooled data (see Results). The bootstrap simulation was done for $C=0.2$ and $C=0.3$.

For a single bootstrap simulation with any data set, sampling units (of $n=15$ leaflets each) were sampled randomly one at a time, with replacement, from a list of observed units, and the cumulative number of diseased leaflets was determined. Sampling ceased when the observed number of diseased leaflets exceeded the model $T_{N}$ or the sample size equaled the size of the data set being sampled. An additional sampling rule imposed was that a minimum of three sampling units must be collected before sampling ceased. Then the moment estimate of $p(\hat{p})$, the standard error of $\hat{p}$, and the achieved $C$ were calculated. A $95 \%$ confidence interval for $p$ was constructed to determine if the interval included the assumed true $p$ (equal to the estimated $p$ for the full data set). The simulation was repeated a total of 100 times for each data set and specified value of $C$. To summarize the results of the simulation, box plots were constructed for achieved $C$ and the difference between (assumed) true $p$ of the data set (estimated $p$ for entire data set) and achieved $p$ after sampling for each of the 18 data sets. The average sample number (ASN) was determined by calculating the mean number of sampling units collected from the 100 simulated sampling bouts for each of the 18 data sets.

\section{RESULTS}

Point-pattern approaches. Distribution analysis. The program BBD successfully calculated maximum likelihood estimates of $p$ and $\theta$ for 50 of the 59 data sets (Table 1 ). Where there was a sufficient number of disease classes for the chi-square goodness-of-fit test to be performed, the frequency distribution of diseased individuals could be described by the beta-binomial distribution in over $75 \%$ of the fields for the 3 years. The binomial distribution described the data in none of the fields. A likelihood ratio statistic (LRS) showed that the frequency distribution of diseased leaflets could be described better by the beta-binomial than by the binomial distribution in 55 of the 59 data sets over 3 years (Table 1). The LRS was not significant $(P>0.05)$ only when mean incidence, determined by $\hat{p}$, was less than 0.05 (Table $1)$.

Figure 2 shows typical transect maps and frequency distributions for four fields with disease incidences ranging from 0.06 to 0.49 and $\hat{\theta}$ of the beta-binomial ranging from 0.16 to 0.62 . The binomial provided a poor fit to all four examples, and the betabinomial provided a very good fit for these, especially for the larger $\hat{\theta}$ 's. One can see the departure of the beta-binomial expected values (open bars) from the binomial expected values (hatched bars) with increasing $\hat{\theta}$ (Fig. 2A to D).

Disease incidence and heterogeneity. Across all 59 data sets, there was a large positive skewness in $\hat{p}$ values (Fig. 3A), with many more small values $(\hat{p}<0.10)$ than large values $(\hat{p}>0.10)$. Mean disease incidence $(\hat{p})$ per data set of leaflets ranged from 0.0009 (i.e., one diseased leaflet in a field) to 0.812 over the 3 years. Considering the locations surveyed in 1998, disease incidence was clearly variable within the state (Fig. 1). The southeastern part of the state (sections 6 and 9) had the highest incidence, with each section having at least one farm with $\hat{p}>0.10$ (Fig. 1). In the north, two of the three sections (2 and 3 ) had no detected diseased leaflets, and the third sector had very low incidence.

Estimates of $\theta$ among the 59 data sets were also positively skewed (Fig. 3B), but less so than for $\hat{p}$ (Fig. $3 \mathrm{~A}$ ). $\hat{\theta}$ ranged from 0 (i.e., binomial) to 1.06 over the 3 years.
Approximately $84 \%$ of the $\hat{\theta}$ 's were significantly greater than 0 according to a $t$ test (11) (data not shown), indicating substantial overdispersion of leaf spot in the commercial fields. Of the eight values of $\hat{\theta}$ that were not significantly different from 0 , all had mean incidence values less than 0.011. Estimated $\theta$ values also were somewhat related to mean incidence (Table 1). With one exception, median $\hat{\theta}$ increased with $\hat{p}$ to the 40 to $60 \%$ class, and then declined.

Binary power law. The binary power law provided an excellent description of the relationship between the observed variance $\left(v_{\mathrm{obs}}\right)$ and variance for a random distribution $\left(v_{\text {ran }}\right)$ on a log scale (Fig. 4A and B). Estimated slopes and intercepts were significantly $(P<0.01)$ greater than one and zero, respectively, for both 1996-97 and 1998 data sets. Thus, there was significant overdispersion of disease incidence across all data sets, and the degree of aggregation depended on incidence (because $\hat{b}>1)(11)$. Most of the points were above the binomial line (Fig. 4A and B), indicating that, although aggregation varied with $\hat{p}$, most data sets exhibited overdispersion, in agreement with results for $\theta$.

The covariance analysis indicated that cultivar, assessment time, region, and year did not significantly affect the intercept or slope $(P>0.05)$. In fact, estimated $\hat{b}$ was the same to two decimal places for the two year categories (1996-97 and 1998). Thus, disease data were pooled over the 3 years, giving estimates of $\ln \left(A_{x}\right)$ and $b$ of 1.29 $(\mathrm{SE}=0.052)$ and $1.23(\mathrm{SE}=0.032)$, respectively.

The binary power law parameter estimates were used to develop prediction curves for the beta-binomial aggregation parameter, $\theta$, based on equation 3 , using the parameter estimates for combined years (Fig. 4C). Therefore, the curve in Figure $4 \mathrm{C}$ was not based on direct curve fitting of the $\hat{\theta}$ values. Although the predicted $\hat{\theta}$ values followed the general trend of $\hat{\theta}$ with $\hat{p}$ (Fig. 4C), there was considerable variation in the individual $\hat{\theta}$ values at any given $\hat{p}$ because the data do not exactly follow a beta-binomial distribution (11).

Table 1. Proportion of data sets with convergence of the maximum likelihood (MLE) procedure, significant likelihood ratio (LRS) test, median estimated beta-binomial $\theta$ parameter, median first-order correlation coefficient $\left(\hat{r}_{1}\right)$, median index of aggregation of the Spatial Analysis by Distance IndicEs (SADIE) procedure $\left(I_{a}\right)$, and proportion of data sets with significant spatial association according to SADIE in relation to categories of leaf spot disease incidence, caused by Mycosphaerella fragariae, for data of 1996 to 1998

\begin{tabular}{|c|c|c|c|c|c|c|c|}
\hline $\begin{array}{l}\text { Disease } \\
\text { incidence class }\end{array}$ & Count & $\begin{array}{c}\text { MLE } \\
\text { convergence }\end{array}$ & $\begin{array}{l}\text { Significant } \\
\text { LRS test }^{\text {b }}\end{array}$ & $\begin{array}{c}\text { Median } \\
\theta\end{array}$ & $\begin{array}{c}\text { Median } \\
\hat{r}_{1} \\
\end{array}$ & $\begin{array}{c}\text { Median } \\
I_{a} \\
\end{array}$ & $\begin{array}{c}\text { Significant } \\
\boldsymbol{P}_{\boldsymbol{a}}{ }^{\mathrm{c}} \\
\end{array}$ \\
\hline $0.00-0.05$ & 29 & $22 / 29$ & $25 / 29$ & 0.107 & 0.027 & 1.24 & $10 / 26$ \\
\hline $0.05-0.10$ & 5 & $4 / 5$ & $5 / 5$ & 0.485 & 0.114 & 1.51 & $1 / 4$ \\
\hline $0.10-0.20$ & 8 & $7 / 8$ & $8 / 8$ & 0.202 & 0.201 & 1.36 & $1 / 7$ \\
\hline $0.20-0.40$ & 8 & $8 / 8$ & $8 / 8$ & 0.212 & 0.275 & 1.84 & $4 / 7$ \\
\hline $0.40-0.60$ & 2 & $2 / 2$ & $2 / 2$ & 0.440 & 0.483 & 3.33 & $2 / 2$ \\
\hline $0.60-0.80$ & 4 & $4 / 4$ & $4 / 4$ & 0.415 & 0.254 & 2.15 & $3 / 4$ \\
\hline $0.80-1.00$ & 3 & $3 / 3$ & $3 / 3$ & 0.295 & 0.129 & 1.10 & $1 / 3$ \\
\hline
\end{tabular}

${ }^{a}$ Classes end with the indicated value and start with next highest incidence value above the listed value.

b Test of the beta-binomial versus the binomial distribution $(P \leq 0.05)$.

${ }^{\mathrm{c}}$ Test for significant spatial aggregation based on the SADIE methodology $(P \leq 0.05)$. 
Geostatistical-type approaches. Spatial autocorrelation. Frequencies of first order autocorrelation coefficients $\left(\hat{r}_{1}\right)$ were slightly skewed to the right (Fig. 3C). Values of $\hat{r}_{1}$ ranged from -0.17 to 0.85 over the 3 years. Median $\hat{r}_{1}$ values increased with $\hat{p}$ up to 0.4 to 0.6 , then declined (Table 1). This relationship can also be seen for the individual data sets in Figure $5 \mathrm{~A}$, although the graph also demonstrates the variability of $\hat{r}_{1}$ values at any $\hat{p}$.

For the 3 years, $42.4 \%$ of the data sets showed significant positive first-order correlations based upon correlation coefficients exceeding the bounds of their ap- proximate $95 \%$ confidence intervals (calculated as $1.645 / \sqrt{ } N$ ). A slightly smaller percentage of second-order autocorrelations $\left(\hat{r}_{2}\right)$ were significant for all 3 years (data not shown). There was no general evidence of a particular type of autoregressive or moving average process (17) for the disease incidence data, even when there was identified spatial autocorrelation. There was not an obvious relationship between $\hat{r}_{1}$ and $\hat{\theta}$ of the beta-binomial distribution (Fig. 5B).

SADIE. The SADIE program was able to calculate distance to regularity $\left(D_{r}\right)$ and distribution of the distance to regularity for randomized arrangements of the samplingunit counts for 53 of the 59 data sets. Frequency distributions of the $53 I_{a}$ values were positively skewed (Fig. 3D). Values of $I_{a}$ ranged from 0.49 to 5.46 over the 3 years. In general, SADIE was able to detect aggregation slightly less often than spatial autocorrelation analysis, with approximately $41.5 \%$ of all data sets being significantly nonrandom $(P<0.05)$. Although there was no anticipation that there would be a relationship between $\hat{p}$ or $\hat{\theta}$ and $I_{a}$, there was a general increase in $I_{a}$ with both $\hat{p}$ and $\hat{\theta}$ (Fig. 5C and D), although there was also high variability. $I_{a}$ was
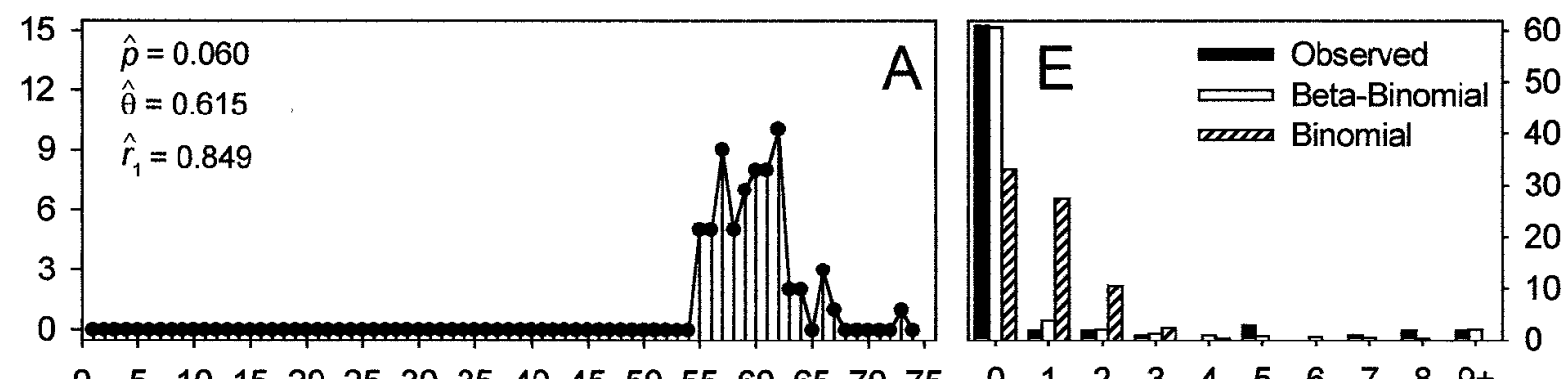

$\begin{array}{llllllllllllllll}0 & 5 & 10 & 15 & 20 & 25 & 30 & 35 & 40 & 45 & 50 & 55 & 60 & 65 & 70 & 75\end{array}$
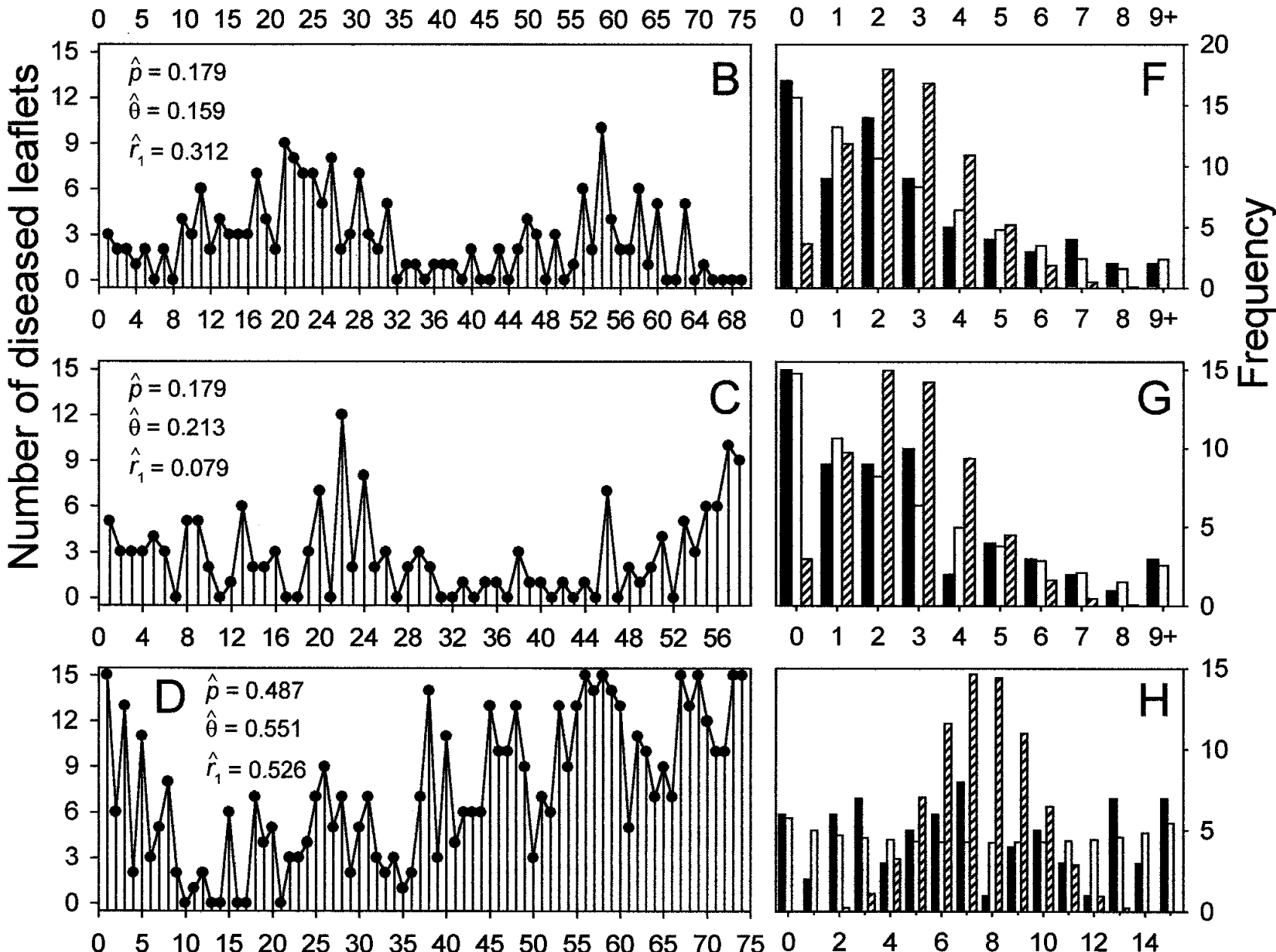

\section{Ordered sampling unit}

\section{Number of diseased leaflets}

Fig. 2. (A to D) Example transect maps of the number of diseased leaflets with leaf spot in individual sampling units (with $n=15$ leaflets per sampling unit) along a randomly chosen row for four example commercial strawberry fields sampled at harvest time. (E to $\mathbf{H})$ Frequency distributions of the observed number of diseased leaflets per sampling unit (solid bars), expected number of diseased leaflets under the beta-binomial distribution (open bars), and expected number of diseased leaflets under the binomial distribution (hatched bars) for the four example fields in parts A to D. 
positively associated with $\hat{r}_{1}$ (Fig. 5E), as expected, since the two statistics both measure similar aspects of spatial arrangement of diseased leaflets.

Sampling curves. Figures $6 \mathrm{~A}$ and $\mathrm{B}$ show sampling curves based on the pooled estimates of the binary power law parameters, with reliability (precision) defined by the coefficient of variation of the mean $(C)$. As the required degree of reliability increased (i.e., as $C$ decreased), larger sample sizes were needed to obtain an estimate of $p$ (Fig. 6A). For the fixed sample size plan, the binomial case (i.e., $A_{x}$ $=1 ; b=1$ ) is shown for comparison (Fig. $6 \mathrm{~B})$. Required sample sizes based on the power law results (i.e., eq. 4; beta-binomial distribution with $\theta$ a function of $p$ ) were always larger than those based on the binomial over the full range of incidences, reflecting the fact that the binary power law line was above the binomial at all incidence values (Fig. 4A and B).

For the sequential sampling plan, the plot of the cumulative number diseased, $T_{N}$ (the stop limits), versus $N$ is shown in Figure $6 \mathrm{C}$. At any given number of sampling units, it takes a larger $T_{N}$ to stop sampling as the required level of precision increases (i.e., as $C$ decreases). For instance, at $N=$ 10 sampling units, one would need $T_{N}=68$ diseased leaflets at $C=0.2$ to cease sam- pling, but would need only $T_{N}=22$ at $C=$ 0.4 . Figure $6 \mathrm{D}$ shows the estimate of incidence at the critical $T_{N}$. Estimates of incidence at the stop limit were always lower as reliability decreased at a given number of sampling units. For the $N=10$ example here, $\hat{p}$ would be 0.45 at the stop limit for $C=0.2$ and 0.15 for $C=0.4$. Of course, with an actual data set, one would not cross the stop limit at the same $N$ for different $C$ 's, but on a value of $N$ that depends on $C$ (Fig. 6C).

To demonstrate the use of these stop limits, estimates of incidence from the four example fields given in Figure 2A to D were determined as if these fields were sampled sequentially. Starting from the first sampling unit and defining the level of reliability as $C=0.3$, one would need to sample 60 units for the example in Figure 2A before crossing the $C=0.3$ limit (shown as the solid circles in Figure 6C). At the sixtieth sampling unit, 39 diseased leaflets were tallied, giving an estimate of $p$ of $39 /(15 \times 60)=0.043$, which is slightly lower than the value of 0.06 determined from the entire data set. For the other example fields (i.e., Fig. 2B to D), estimates of $p$ were $0.184(N=17), 0.21(N=13)$, and $0.75(N=3$; which was chosen as the arbitrary minimum number of units to sample) when $T_{N}$ crossed the stop limit.
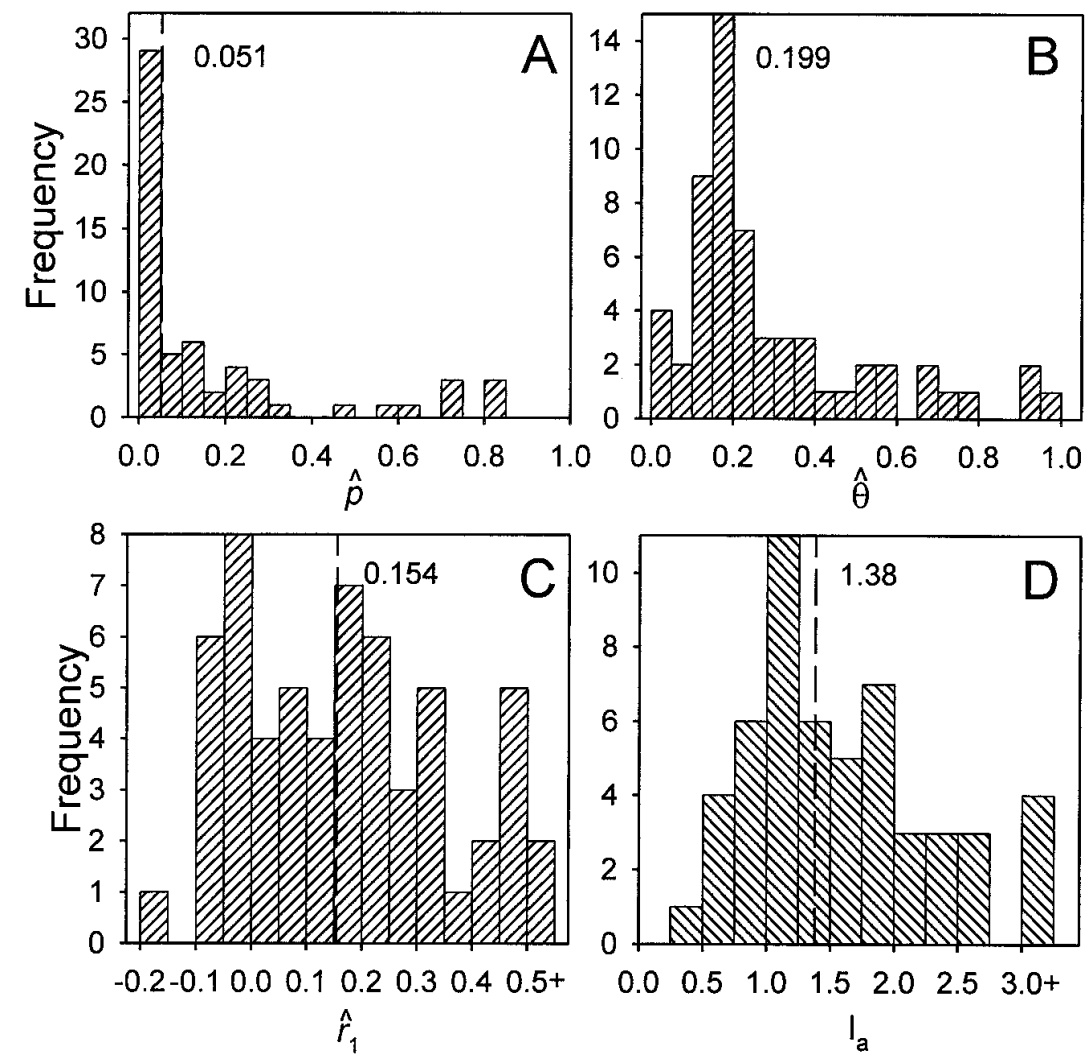

Fig. 3. Frequency distributions of (A) estimated beta-binomial parameter $\hat{p}$ (mean incidence per field), (B) estimated beta binomial aggregation parameter $\hat{\theta}$, (C) first-order autocorrelation coefficient $\left(\hat{r}_{1}\right)$, and (D) Spatial Analysis by Distance IndicEs (SADIE) index of aggregation $I_{a}$. Frequency distributions are based on 59 data sets of the incidence of leaf spot collected from 1996 to 1998 from commercial strawberry fields. Vertical dashed line represents the median value of the indicated statistic; numerical value is given on the graph.

The estimates of $p$ based on sequential estimation were either higher or lower than the estimates obtained from our standard (full data set) sampling protocol (see $\hat{p}$ values in Figure 2), indicating a lack of bias, and calculated $C$ was always less than 0.3 .

Sequential sampling evaluation. Figures 7A and B show that the achieved coefficient of variation was generally close to or below the prespecified value of $C$. This was especially true when $C=0.3$ was specified. Achieved $C$ was most distant from the prespecified $C$ at lower incidences, especially for $C=0.2$. This occurred because sampling ceased before the
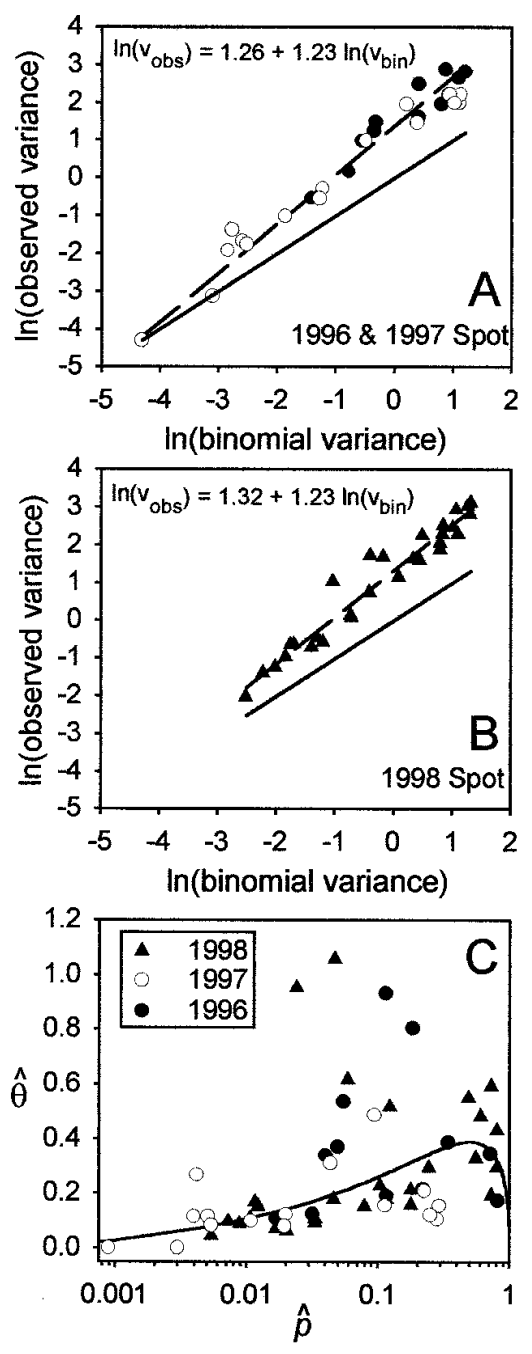

Fig. 4. Relationship between the logarithm of the observed variance $\left[\ln \left(v_{\text {obs }}\right)\right]$ and logarithm of the theoretical variance for a binomial distribution $[\ln (n \hat{p}[1-\hat{p}])]$ for incidence $(\hat{p})$ of strawberry leaf spot in (A) 1996 and 1997 and (B) 1998. Dashed lines represent the least squares fit to the 59 data sets and the solid line represents the binomial line (i.e., with intercept of 0 and slope of 1). (C) Relationship between estimated $\theta$ of the beta-binomial and $\hat{p}$ for 1996 to 1998 . Solid line represents predicted $\theta$ (eq. 3 ) based on least-squares estimates of the binary power law parameters. Years are labeled with different symbols. 
cumulative number of diseased leaflets exceeded the model $T_{N}$; that is, cumulative number diseased was less than $T_{N}$ when $N$ equaled the actual number of sampling units in the full data set. Figure 6A shows the average sample number (ASN) for the 100 bootstrap simulations superimposed on the predicted number of sampling units needed to achieve a prespecified level of $C$ for $C=0.2$ and $C=0.3$. At lower incidences, the number of sampling units collected was less than what is needed in order to estimate $p$ with the desired level of reliability. Again, this is because sampling ceased when sample size equaled the size of the data set, and not when desired $C$ was reached. Additionally, the number of sampling units collected exceeded model prediction at higher incidences due to the sampling rule imposed. In these cases, the number of diseased leaflets somewhat exceeded model $T_{N}$ before the third sampling unit was collected. This resulted in achieved $C$ being less than the prespecified $C$ (Fig. 7A and B).

In approximately $93 \%$ of the evaluated data sets, achieved $p$ was not significantly different from (assumed) true $p(=\hat{p}$ for the full data) according to $t$ tests (13). Figures $7 \mathrm{C}$ and D show that the median difference between true and achieved $p$ was generally around zero for both $C=$ 0.2 and $C=0.3$. The variability of the difference increased with increasing incidence and was generally larger for $C$ $=0.3$, as expected (13).

\section{DISCUSSION}

The spatial pattern of leaf spot incidence was characterized over 3 years at several
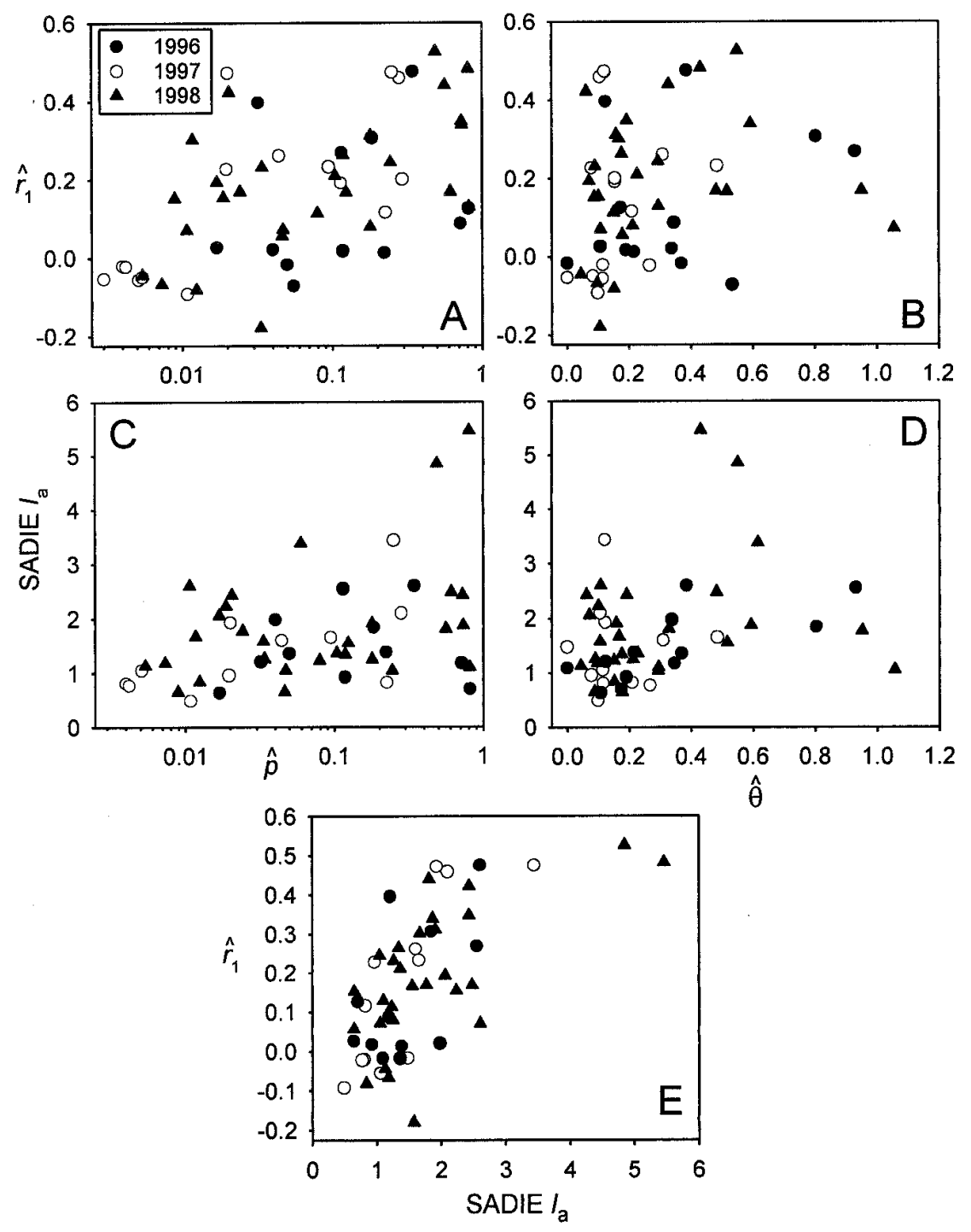

Fig. 5. Relationships between (A) estimated beta-binomial parameter $\hat{p}$ (mean disease incidence) and first-order spatial autocorrelation coefficient $\left(\hat{r}_{1}\right),(\mathbf{B})$ estimated beta-binomial aggregation parameter $\hat{\theta}$ and $\hat{r}_{1}$, (C) $\hat{p}$ and Spatial Analysis by Distance IndicEs (SADIE) index of aggregation $\left(I_{a}\right)$, (D) $\hat{\theta}$ and $I_{a}$, and (E) $I_{a}$ and $\hat{r}_{1}$. Each point represents a single field assessment of the incidence of strawberry leaf spot from 1996 to 1998 . To maintain a consistent scale for $\hat{p}$, two low-incidence points were omitted from $\mathbf{A}$ and $\mathbf{C}$.

commercial strawberry farms in Ohio. In general, spatial heterogeneity was well described by estimated $\theta$ of the beta-binomial distribution; estimates indicated significant spatial aggregation of disease incidence. That is, there was a positive correlation of disease status of leaflets within sampling units in $84 \%$ of the data sets. The degree of heterogeneity varied with incidence according to the binary power law, thus justifying fitting the betabinomial distribution to the data. Estimated $b$ of the power law was greater than 1 , which indicates that $\hat{\theta}$ was a function of $\hat{p}$ (Table 1, Fig. 4C). After accounting for the influence of disease incidence on heterogeneity through the binary power law, cultivar, year, assessment time, or region did not significantly influence the degree of heterogeneity as indicated by analysis of covariance.

About $40 \%$ of the data sets showed significant spatial aggregation according to the SADIE procedure and autocorrelation analysis, indicating that disease clusters extended beyond the borders of the sampling units in these cases (20). Degree of spatial association of disease incidence between sampling units $\left(\hat{r}_{1}, I_{a}\right)$ generally increased with incidence (Fig. 5A and $\mathrm{C}$ ) up to $\hat{p}=0.4$ to 0.6 and then declined (Table 1). However, there were not many data sets in this middle category to clearly identify this incidence as the optimum for degree of spatial association. The SADIE procedure identified significant spatial association slightly less frequently than did the first-order autocorrelation analysis. However, the close agreement in these results (Fig. 5E) suggests that heterogeneity of disease incidence (measured by $\hat{\theta}$ ) did not greatly affect measurements of spatial associations among sampling units (20).

In the only other previous use of SADIE for disease incidence collected with cluster sampling, SADIE detected significant spatial associations somewhat more frequently than did first-order autocorrelation analysis (20). However, SADIE was used in the previous study to test for departures from randomness (aggregation and regularity) rather than to test just for aggregation (as in the current study). Testing specifically for aggregation is a more powerful test for detecting aggregation than testing for aggregation under the general alternative of randomness (i.e., a one-sided versus a twosided test). Because it is well documented that disease tends to occur in clumps $(2,13,20)$, it is more practical to directly test for aggregation than for unspecific nonrandomness. However, when SADIE and autocorrelation analysis were both conducted as two-sided tests in the current study, SADIE detected significant associations somewhat more frequently than autocorrelation analysis (33\% versus 29\%) (data not shown). Only two data sets in the leaf spot study showed significant regular 
(uniform) patterns according to SADIE; these had low estimated $p$ values of 0.0042 and 0.0108 . In principle, the SADIE methodology may be more appropriate for the analysis of binary or count data than spatial autocorrelation analysis because of the discrete nature of the data and frequent occurrence of zeros $(1,16)$.

Using the same sampling methodology as described here, we recently described the spatial pattern of Phomopsis leaf blight on strawberry (20). Leaf blight and spot are typically found together in a field and sometimes on the same leaflets, and both pathogens overwinter in infected strawberry tissue. In general, clumps of leaf blight were smaller (as determined by the magnitude of spatial correlation $\left.\left[I_{a}, \hat{r}_{1}\right]\right)$, and the degree of heterogeneity $(\hat{\theta})$ was lower than for spot. Although it has been stated that conidia of both of these leafspotting pathogens are splash dispersed (9), there is no experimental evidence of this for $R$. brunnea. Spatial heterogeneity similar to that of leaf spot has been found for some other wind-dispersed pathogens (12). The conidia and fruiting structure of $R$. brunnea are morphologically similar to several fungi that are known to be wind disseminated (5), and moreover, other Ramularia spp. are reported to be wind disseminated (14). An alternative explanation for the differences is that wind dispersal of ascospores of the leaf spot pathogen in the spring has a large influence on the final spatial pattern in the summer. Our results on the spatial patterns of leaf spot incidence indicated that controlled studies are clearly needed to determine the dominant spore dispersal mechanism for this pathogen.

The impact of spatial heterogeneity can be clearly seen in the calculated sampling curves developed for estimating incidence of leaf spot with desired reliability. Based on the binary power law results, larger sample sizes were needed compared with a random (i.e., binomial) distribution to reliably estimate mean incidence. If one were to sample and ignore the effects of heterogeneity, i.e., use sampling curves based on the binomial distribution, $\hat{p}$ would be estimated with a false sense of reliability (if one incorrectly calculated the standard error of $\hat{p}$ based on the binomial formula), or $\hat{p}$ would be estimated with lower reliability than desired (if one calculated standard error of $\hat{p}$ correctly after first using the incorrect sampling curves). Although one does not know the value of $p$ before sampling occurs, a rough estimate is satisfactory if $p$ is not small. For example, with
$C=0.3$, required $N$ is $\sim 26$ when $p=0.10$ and $\sim 8$ when $p=0.30$. Thus, choosing $N=$ 20 is quite reasonable. However, at $p<$ 0.05 (at the same $C$ ), the variation in $N$ can be large. Required $N$ is $\sim 50$ at $p=0.05$, $\sim 200$ at $p=0.01$, and $\sim 300$ at $p=0.005$. Thus, selection of $N$ during a field sampling can be very beneficial.

The sequential sampling plan, demonstrated in Figure 6C, can save considerable time, since the number of sampling units collected is conditioned on the level of incidence in the field. When disease incidence is high (say, $p>0.40$ ), sampling will terminate rapidly (i.e., at small $N$; Fig. 6D). However, when incidence is low (say, $p<0.10$; e.g., Fig. 2A), a large number of sampling units may need to be observed before a reliable estimate can be made. This can be seen in Figure 6A, where the ASN equaled the size of the data set for sets 1 and 2 (points corresponding to the two lowest $\hat{p}$ values) when $C=0.3$ and for sets 1 to 4 when $C$ $=0.2$. In these instances, the data sets were too small according to model predictions to reliably estimate $p$; this is confirmed in Figure 7A and B. Sequential sampling plans, such as the one developed here, have not been used much in plant pathology $(2,13)$, but they have
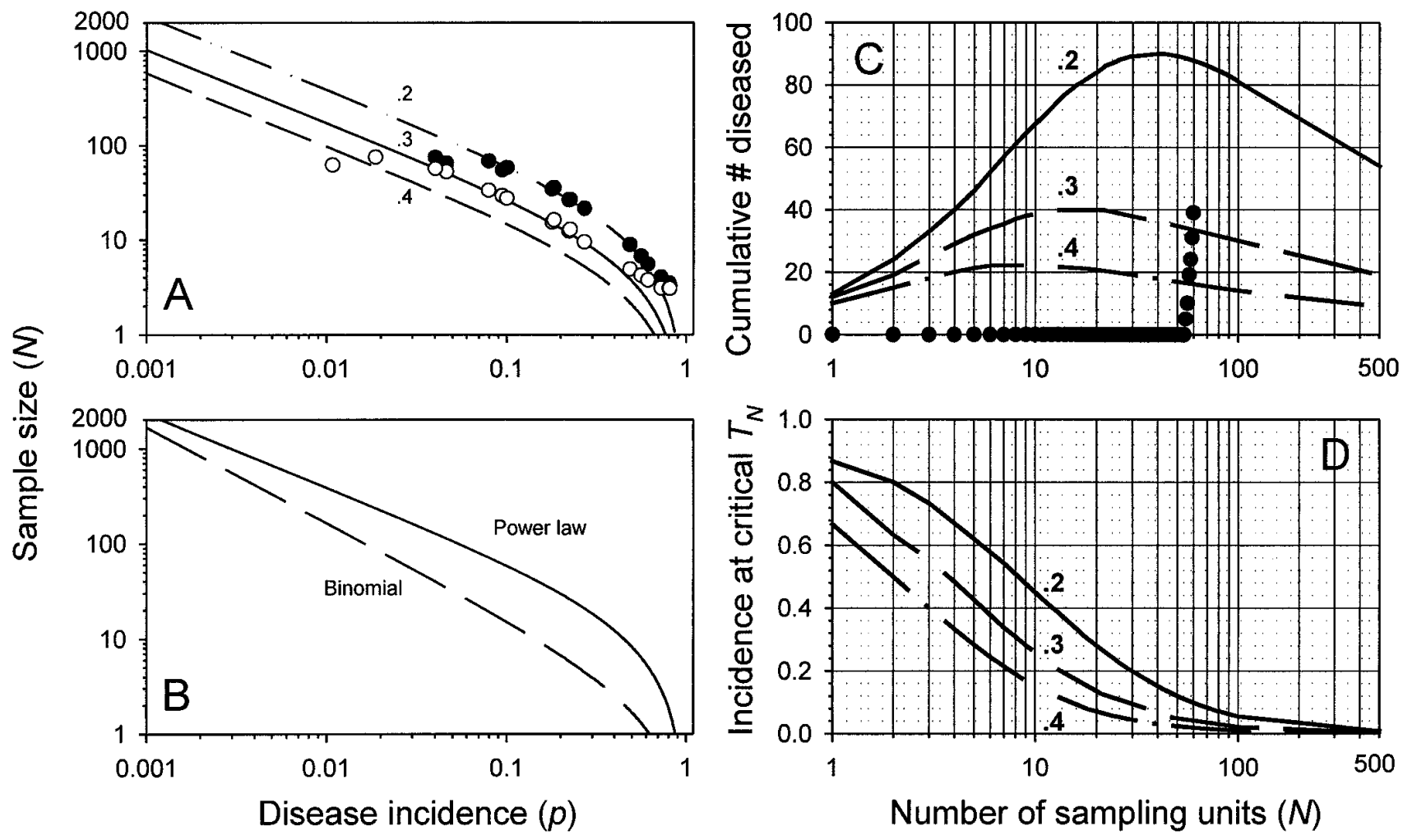

Fig. 6. Fixed and sequential sampling curves for estimating mean disease incidence $(\hat{p})$ of strawberry leaf spot with coefficients of variation of the mean $(C)$ of $0.2,0.3$, and 0.4. (A) Number of sampling units based on the binary power law (eq. 4$)$ with $b=1.23$ and $a=0.45\left(a=A_{x} n^{b-2}\right)$ for fixed sample size at three labeled values of $C$. Superimposed is the average sample number (ASN) for the evaluation of 100 bootstrap simulations of sequential sampling for $C=0.3$ (open circles) and $C=0.2$ (solid circles). (B) Number of sampling units versus $p$ for the binomial $(a=1 / 15=0.067 ; b=1)$ and power law $(a=$ $0.45 ; b=1.23$ ) cases with $C=0.2$. (C) Cumulative number of diseased leaflets $\left(T_{N}\right)$ versus number of sampling units based on the binary power law (eq. 5) with $b=1.23$ and $a=0.45$ and three $C$ values. (D) Mean disease incidence at critical $T_{N}$ (when cumulative number diseased crosses $T_{N}$ curve in Figure 7C); mean disease incidence is estimated by $T_{N} / n N$. 

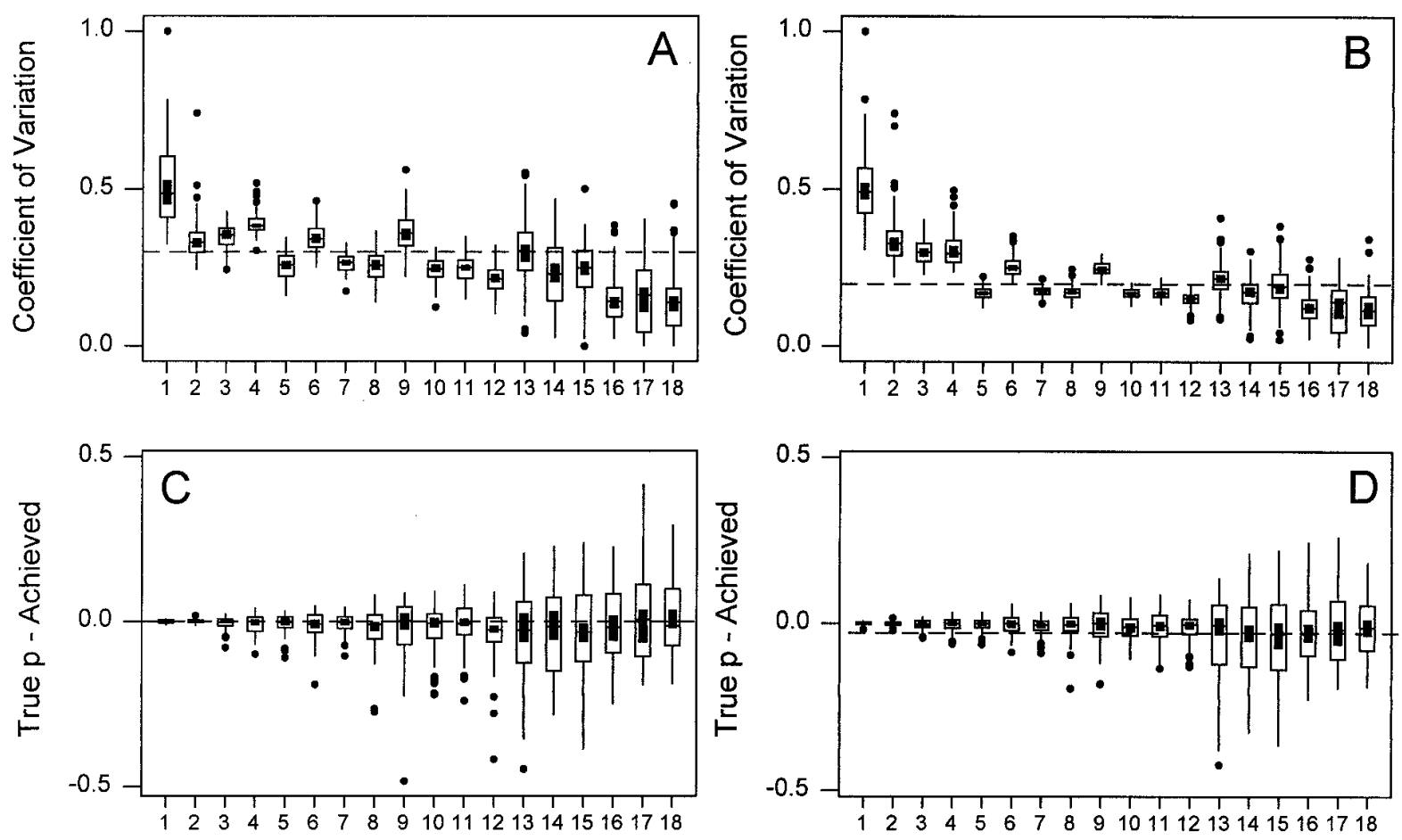

\section{Leaf Spot Data Sets}

Fig. 7. Box plots of the achieved coefficient of variation of estimated disease incidence $(\hat{p}), C$, and the difference of the true value of $p$ and $\hat{\mathrm{p}}$ from 100 bootstrap samplings of 18 leaf spot data sets based on use of equation 5. Data sets are arranged in increasing order of $\hat{p}$, such that data sets 1 to 3 correspond to $0.01 \leq \hat{p}<0.05$, sets 4 to 6 correspond to $0.05 \leq \hat{p}<0.1$,; sets 7 to 9 correspond to $0.1 \leq \hat{p}<0.2$, sets 10 to 12 correspond to $0.2 \leq \hat{p}<0.4$, sets 13 to 15 correspond to $0.4 \leq \hat{p}<0.65$, and sets 16 to 18 correspond to $\hat{p} \geq 0.65$. Preselected values of $C$ are 0.3 for (A and C) and 0.2 for (B and D). Box plots show the median, middle $50 \%$ of data (open box), $95 \%$ confidence interval for median (solid bar inside box, based on sign test), "whiskers" (vertical straight lines) that extend to the lowest and highest observations that are still within the region defined by lower and upper limits, and outliers (solid circles) beyond the whiskers. Lower limit is defined as Q1 - 1.5·(Q3 - Q1), and upper limit is defined as Q3 + 1.5·(Q3 - Q1), where Q1 and Q3 are the first and third quartiles of the data.

great potential for reducing the time and cost of sampling for the purpose of precisely estimating spatially aggregated plant disease incidence.

\section{LITERATURE CITED}

1. Bellehumer, C., and Legendre, P. 1998. Multiscale sources of variation in ecological variables: Modeling spatial dispersion, elaborating sampling designs. Landscape Ecol. 13:15-25.

2. Campbell, C. L., and Madden, L. V. 1990. Introduction to Plant Disease Epidemiology. John Wiley \& Sons, New York.

3. Collett, D. 1991. Modelling Binary Data. Chapman \& Hall, London.

4. Ellis, M. A. 1998. Strawberry leaf spot diseases. Ohio State University Extension Fact Sheet. HYG-3015-95.

5. Fitt, B. D. L., McCartney, H. A., and Walklate, P. J. 1989. The role of rain dispersal of pathogen inoculum. Annu. Rev. Phytopathol. 27:241-270.

6. Hughes, G., and Madden, L. V. 1992. Aggregation and incidence of disease. Plant Pathol. 41:657-660.

7. Hughes, G., and Madden, L. V. 1993. Using the beta-binomial distribution to describe aggregated patterns of disease incidence. Phytopathology 83:759-763.

8. Hughes, G., Madden, L. V., and Munkvold, G. P. 1996. Cluster sampling for disease incidence data. Phytopathology 86:132-137.

9. Maas, J. L., ed. 1984. Compendium of Strawberry Diseases. American Phytopathological Society, St. Paul, MN.

10. Madden, L. V., and Hughes, G. 1994. BBDcomputer software for fitting the beta-binomial distribution to disease incidence data. Plant Dis. 78:536-540.

11. Madden, L. V., and Hughes, G. 1995. Plant disease incidence: Distributions, heterogeneity, and temporal analysis. Annu. Rev. Phytopathol. 33:529-564.

12. Madden, L. V., Hughes, G., and Ellis, M. A. 1995. Spatial heterogeneity of the incidence of grape downy mildew. Phytopathology 85:269-275.

13. Madden, L. V., Hughes, G., and Munkvold, G. P. 1996. Plant disease incidence: Inverse sampling, sequential sampling, and confidence intervals when observed mean incidence is zero. Crop Prot. 15:621-632.

14. Nyvall, R. F. 1989. Field Crop Diseases
Handbook. 2nd ed. Van Nostrand Reinhold, New York.

15. Perry, J. N. 1995. Spatial aspects of animal and plant distribution in patchy farmland habitats. Pages 221-242 in: Ecology and Integrated Farming Systems. D. M. Glen, M. P. Greaves, and H. M. Anderson, eds. John Wiley \& Sons, London.

16. Perry, J. N. 1998. Measures of spatial pattern for counts. Ecology 79:1008-1017.

17. Reynolds, K. M., and Madden, L. V. 1988 Analysis of epidemics using spatio-temporal autocorrelation. Phytopathology 78:240-246.

18. Reynolds, K. M., Madden, L. V., and Ellis, M. A. 1988. Spatio-temporal analysis of epidemic development of leather rot of strawberry. Phytopathology 78:246-252.

19. Taylor, L. R. 1961. Aggregation, variance and the mean. Nature (Lond.) 189:732-735.

20. Turechek, W. W., and Madden, L. V. 1999. Spatial pattern analysis of strawberry leaf blight in perennial production systems. Phytopathology 89:421-433.

21. Williams, D. A. 1975. The analysis of binary responses from toxicological experiments involving reproduction and teratogenicity. Biometrics 31:949-952. 